\title{
Outcomes of advance care directives after admission to a long-term care home: DNR the $\mathrm{DNH}$ ?
}

Rhéda Adekpedjou', George A. Heckmann ${ }^{2,3^{*}}$, Paul C. Hébert ${ }^{1}$, Andrew P. Costa ${ }^{4}$ and John Hirdes ${ }^{2}$

\begin{abstract}
Background: Residents of long-term care homes (LTCH) often experience unnecessary and non-beneficial hospitalizations and interventions near the end-of-life. Advance care directives aim to ensure that end-of-life care respects resident needs and wishes.
\end{abstract}

Methods: In this retrospective cohort study, we used multistate models to examine the health trajectories associated with Do-Not-Resuscitate (DNR) and Do-Not-Hospitalize (DNH) directives of residents admitted to LTCH in Ontario, Alberta, and British Columbia, Canada. We adjusted for baseline frailty-related health instability. We considered three possible end states: change in health, hospitalization, or death. For measurements, we used standardized RAI-MDS 2.0 LTCH assessments linked to hospital records from 2010 to 2015.

Results: We report on 123,003 LTCH residents. The prevalence of DNR and DNH directives was 71 and 26\% respectively. Both directives were associated with increased odds of transitioning to a state of greater health instability and death, and decreased odds of hospitalization. The odds of hospitalization in the presence of a DNH directive were lowered, but not eliminated, with odds of 0.67 (95\% confidence interval 0.65-0.69), 0.63 (0.61-0.65), and 0.47 (0.430.52) for residents with low, moderate and high health instability, respectively.

Conclusion: Even though both DNR and DNH orders are associated with serious health outcomes, DNH directives were not frequently used and often overturned. We suggest that policies recommending DNH directives be re-evaluated, with greater emphasis on advance care planning that better reflects resident values and wishes.

Keywords: Advance care planning, Long-term care homes, Nursing homes, interRAl

\section{Background}

In Canada, long-term care homes (LTCH) designate residential facilities that provide 24 -h nursing and personal support to people who can no longer live independently in the community [1]. The period following LTCH admission is one during which many residents experience greater health instability, are hospitalized, or die [2]. LTCH residents are at increased risk of acute

\footnotetext{
*Correspondence: ggheckma@uwaterloo.ca

${ }^{2}$ School of Public Health Sciences, University of Waterloo, 200 University

Avenue West, Waterloo, Ontario N2L 3G1, Canada

Full list of author information is available at the end of the article
}

care utilization, with about one third being hospitalized yearly, during which they often undergo multiple diagnostic tests and procedures [3]. Many of these acute care visits and treatments are potentially avoidable, frequently unnecessary, and often discordant with a resident's care wishes and preferences [4]. Indeed, older adults often focus on quality of life, function, and symptom control, and most prefer to die at home rather than in hospital [5].

Advance care planning is a process that supports residents, their families, and health care professionals make decisions about future medical care that reflect resident goals, values and preferences [6]. If done well, advance 
care planning protects resident autonomy at the end-oflife, even in the presence of cognitive impairment $[6,7]$. In Canada, LTCH homes usually codify the outcomes of advance care planning in medical orders such as DoNot-Hospitalize (DNH) and Do-Not-Resuscitate (DNR), though policies and regulations surrounding related care processes vary among individual Canadian provinces and territories $[8,9]$. Such directives are associated with fewer hospitalisations and hospital days, death in acute care, and use of life-sustaining treatments such as cardiopulmonary resuscitation, and with a marginal impact on survival time. They are also associated with an increased probability of dying in the $\mathrm{LTCH}[7,8]$.

Recent population-based data from in Ontario, Canada LTCH shows that while frailty-related health instability of newly admitted residents was associated with the presence of DNR and DNH directives, this very instability remained associated with an increased risk of hospitalisation during the first 90 days after admission $[3,8]$. These findings suggest that while the presence of a DNR or DNH directive identifies more unstable and frailer LTCH residents potentially less likely to benefit from hospitalization, the $\mathrm{DNH}$ directive may be challenging to implement in actual practice. It is thus important to understand how admission health status and directives such as DNR and DNH relate to resident transitions to different health states, particularly during the period following admission to $\mathrm{LTCH}$, in order to ensure that residents receive care that is concordant with their wishes. The aim of the present study is to understand the association of DNR and DNH directives, adjusted for baseline health instability, on subsequent transitions to different health states for newly admitted residents to LTCH in three Canadian provinces.

\section{Methods}

\section{Study design}

We conducted a retrospective cohort study of newly admitted LTCH residents from January 1st 2010 to March 31st 2015 in British Columbia, Alberta, and Ontario, Canada. We obtained approval from the University of Waterloo Office of Research Ethics (ORE\# 18228). We complied with the Declaration of Helsinki. We applied RECORD (Reporting of Studies Conducted Using Observational Routinely Collected Health Data) and STROBE (Strengthening the Reporting of Observational Studies in Epidemiology) guidelines to report our findings $[10,11]$.

\section{Data sources}

We used the following administrative data sets (a) the Complex Continuing Care Reporting System (CCRS) based on the interRAI Resident Assessment
Instrument-Minimum Data Set (RAI-MDS) 2.0 for $\mathrm{LTCH},(\mathrm{b})$ the Discharge Abstract Database (DAD) which tracks acute hospital admissions and (c) the National Ambulatory Care Reporting System (NACRS) which tracks emergency department visits. We obtained these from the Canadian Institute for Health Information, which exerts strict controls over data quality, validity, and integrity of each of these data sources [12-14]. These data sets were linked using individual resident's unique Provincial health insurance identifiers.

The RAI-MDS 2.0 is a standard assessment mandated for use in 8 of 13 Canadian provinces and territories. The instrument is administered by trained assessors within 14days of admission, with follow-up assessments completed every 90 days, or earlier in the event of major clinical changes [1]. The instrument contains over 300 clinical variables to support care planning, outcome measurement, quality improvement and resource allocation, and multiple embedded scales such as the Activities of Daily Living Hierarchy Scale, the Instrumental Activities of Daily Living Scale [15], the Changes in Health, End-Stage Disease, Signs, and Symptoms Scale (CHESS) [16, 17], the Depression Rating Scale [18, 19], and the Cognitive Performance Scale [20-22]. The presence of DNR and $\mathrm{DNH}$ directives in the resident chart is also recorded in the RAI-MDS 2.0. Reliability and validity of the RAI 2.0 and associated items, scales and algorithms have been well-established by several international studies [23-25]. Likewise, the reliability of that data captured in the DAD and NACRS has also been established $(2,3)$.

\section{Study cohort}

We used data from January 1st 2010 to March 31st 2015 targeting LTCH residents in Ontario, Alberta and British Columbia. These provinces were included because their RAI-MDS 2.0 information systems were well-established during our study period. We included linked residents (i.e., whose information was contained in multiple data sources) if they had (a) at least 1 follow-up assessment at 90 days after admission to the LTCH (b) or a date of discharge or death within 90 days of admission to the LTCH. The initial cohort included 373,760 residents from across Canada (see Fig. 1). We excluded residents if: 1) gender was "Other" because small cell sizes would create privacy concerns $(n=36), 2)$ the assessment or entry date was missing, 3) CHESS scale assessment information was incomplete $(n=73), 4)$ the first available assessment is not the admission to LTCH assessment for the resident and which antedates the study period $(n=182,265), 5)$ the stay in a LTCH was purely short stay to provide the family caregiver with temporary respite $(n=18,354), 6)$ the resident was aged less than 65 years old at time of initial assessment $(n=9556), 7)$ first assessments were after 


\section{All LTCH residents in CCRS} from January $1^{\text {st }} 2014$ to March $31^{\text {st }} 2015: \mathrm{n}=373,760$

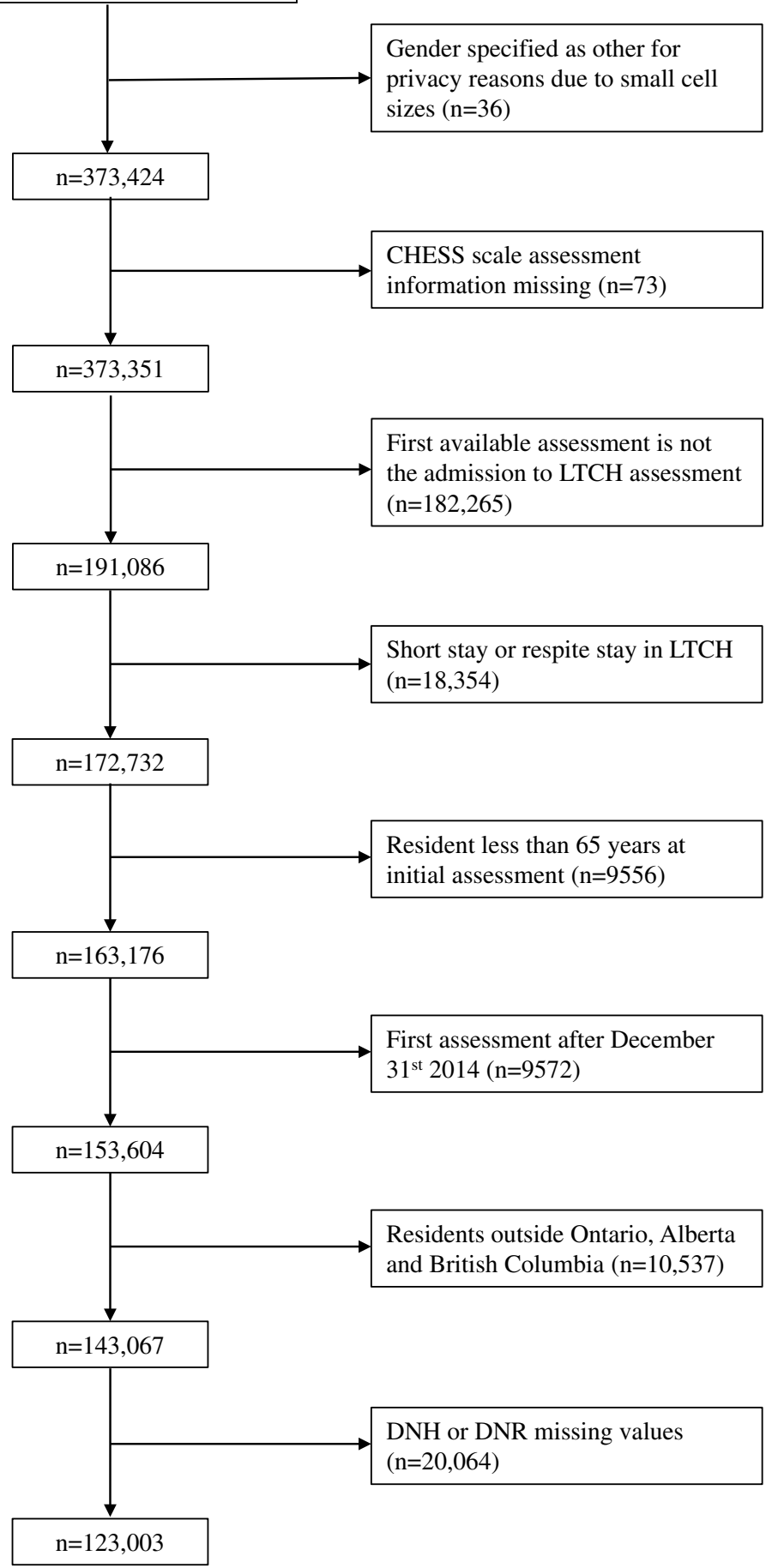

Fig. 1 Derivation of the analytical cohort 
Dec 31st 2014 to allow for 90 day follow-up to March 31st $2015(n=9572), 8)$ residents were from provinces other than Ontario, Alberta and British Columbia $(n=10,537)$, or 9) $\mathrm{DNH}$ or DNR values were missing on the initial assessment $(n=20,064)$.

\section{Outcomes of interests}

We tracked five types of transitions in the 90-day following admission to the LTCH: 1) transition to a different state health instability; 2) discharge from the LTCH; 3) transfer to other care settings (e.g., other LTCH, hospice, home); 4) hospitalization, and 5) death. Health instability was measured with the CHESS (see Table 1). The CHESS scale rates health risk related to acutely decompensated frailty with scores from 0 (low instability) to 5 (high instability), and higher scores predict hospitalization and mortality among LTCH residents $[16,17]$. We defined 3 states of health instability corresponding to low (CHESS 0 ), medium (CHESS 1-2) and high (CHESS 3-5) instability $[16,17]$. We used 0 as a distinctive first state (rather than $0-1$ ) because 0 designates the absence of any indicators of health instability, a clinically relevant break point. We ascertained hospitalizations, death, and discharge to another setting using the relevant CCRS, DAD, and NACRS variables [12-14].

\section{Statistical methods}

We used methods previously applied to data of this type by Cook and colleagues and summarize them briefly here [26, 27]. Multistate transition analysis, based on discrete time nonhomogeneous Markov chain models, is a powerful tool to examine longitudinal changes in multiple health status outcomes over time and identify factors that influence these changes, thus allowing for the examination of competing risks in models where different outcomes (e.g., death and hospital admission) may be affected by similar risk factors [27]. Sample description with descriptive statistics is an intermediate step of Multistate transition analysis. We designated assessment times as Time 1 (T1) for the baseline admission RAI-MDS 2.0 assessment and as Time 2 (T2) for the first follow-up assessment at 90 days. Exposure variables were DNR and DNH directives based on the T1 RAI-MDS 2.0 assessment: 1) DNH present vs. not present and 2)
DNR present vs. not present. Outcome variables were i) change in state of health instability at $\mathrm{T} 2$ versus $\mathrm{T} 1$, ii) location of care at T2 if discharged from the LTCH (hospital, another setting or home) and iii) death by T2. Discharge destinations and death are considered absorbing states, because transitioning to one of these states defines the end of the particular care episode in the LTCH. Residents remained in the model until the end of T2 or their first transition out of the LTCH.

We adjusted outcomes for age, sex, marital status, Activities of Daily Living Hierarchy Scale score, Cognitive Performance Scale score, diagnosis (binary variables for chronic obstructive pulmonary disease, pneumonia, diabetes, arthritis, renal failure, urinary tract infection, Alzheimer's dementia and related dementias, heart failure, cancer and depression), day of stay, functional improvement potential, facility size, and province. We chose covariates on the basis of their expected associations with one or more of the outcomes states we modelled $[3,28,29]$. We only retained covariates that had significant associations with at least one outcome of interest in the models we used. We used odds ratio as a measure of association. To examine the degree of variation of this association according to illness severity, we looked at the computed odds ratios within the three levels of health instability as defined above: low, medium and high health instability. Analyses were performed using SAS Windows Server, version 9.4 (SAS Institute, Inc., Cary, NC).

\section{Results \\ Baseline characteristics of $\mathrm{LTCH}$ residents}

The final sample consisted of 123,003 LTCH residents (Table 2) who underwent 162,045 transitions. Overall, about $64 \%$ were women, and about $90 \%$ of patients were older than 75 years. Across Canada, $71 \%$ of residents had DNR directives, with the greatest proportion in Alberta at $83 \%$ and the lowest in Ontario at $70 \%$. Over a quarter of residents had DNH directives with the highest proportion in Alberta (31\%) and the lowest proportion in British Columbia (24\%). Among residents with a DNR directive, $34.7 \%$ also had a DNH directive. Only $3.3 \%$ of residents with a DNH directive did not have a DNR directive.

Table 1 The CHESS Scale

\footnotetext{
The CHESS scale: The RAI MDS 2.0 is completed by trained assessors. These make use of all possible sources of information to assess a LTCH resident. CHESS scale scores range from 0 (no health instability) to 5 (very high health instability) based on the following:

- 1 point each for recent (within 2 weeks) decline in Activities of Daily Living and Cognitive Performance;

- Up to 2 points for the presence of any of the following: insufficient fluid intake, peripheral edema, shortness of breath, vomiting, weight loss, decrease in food eaten;

- 1 point based on the clinical judgement of a physician that a resident has at most 6 months to live.
} 
Table 2 Distribution of baseline co-variates at time of admission to LTCH by DNH and DNR Status ( $n=123,003$ residents)

\begin{tabular}{|c|c|c|c|c|c|c|c|c|}
\hline \multirow[t]{2}{*}{ Covariate } & \multirow[t]{2}{*}{ Domain } & \multicolumn{3}{|c|}{ Do Not Hospitalize } & \multicolumn{3}{|c|}{ Do Not Resuscitate } & \multirow[t]{2}{*}{ All Residents (\%) } \\
\hline & & Not Present (\%) & Present (\%) & $\begin{array}{l}\mathrm{X}^{2} \\
p \text { value }\end{array}$ & Not Present (\%) & Present (\%) & $\begin{array}{l}\mathrm{X}^{2} \\
p \text { value }\end{array}$ & \\
\hline \multirow[t]{4}{*}{ Age Group } & $65-74$ & $10,402(78.3)$ & $2889(21.74)$ & $<.0001$ & $5602(42.2)$ & $7689(57.8)$ & $<.0001$ & 13,291 (10.8) \\
\hline & $75-84$ & $33,504(76.9)$ & $10,082(23.1)$ & & $14,493(33.3)$ & $29,093(66.7)$ & & $43,586(35.4)$ \\
\hline & $85-94$ & $42,105(73.1)$ & $15,503(26.9)$ & & $14,270(24.8)$ & $43,338(75.2)$ & & $57,608(46.8)$ \\
\hline & $95+$ & $5664(66.5)$ & $2854(33.5)$ & & $1451(17.0)$ & 7067 (83.0) & & $8518(7.0)$ \\
\hline \multirow[t]{2}{*}{ Gender } & Female & $58,889(74.2)$ & $20,529(25.8)$ & $<.0001$ & $22,410(28.2)$ & $57,008(71.8)$ & $<.0001$ & $79,418(64.6)$ \\
\hline & Male & $32,786(75.2)$ & $10,799(24.8)$ & & $13,406(30.8)$ & $30,179(69.2)$ & & 43,585 (35.4) \\
\hline \multirow[t]{2}{*}{ Marital Status } & Not married & $65,784(74.1)$ & $22,942(25.9)$ & $<.0001$ & $24,884(28.1)$ & $63,842(71.9)$ & $<.0001$ & $88,726(72.1)$ \\
\hline & Married & $25,891(75.5)$ & $8386(24.5)$ & & $10,932(31.9)$ & $23,345(68.1)$ & & $34,277(27.9)$ \\
\hline \multirow[t]{3}{*}{ Province } & $A B$ & $8541(68.4)$ & $3943(31.6)$ & $<.0001$ & $2155(17.3)$ & $10,329(82.7)$ & $<.0001$ & $12,484(10.2)$ \\
\hline & $\mathrm{BC}$ & $13,276(76.0)$ & $4186(34.0)$ & & $4856(27.8)$ & $12,606(72.2)$ & & $17,462(14.2)$ \\
\hline & ON & $69,858(75.1)$ & 23,199 (24.9) & & $28,805(31.0)$ & $64,252(69.0)$ & & $93,057(75.6)$ \\
\hline \multirow[t]{16}{*}{ Diagnoses } & COPD & $15,008(73.8)$ & $5339(26.2)$ & 0.0058 & $5403(26.6)$ & $14,944(73.5)$ & $<.0001$ & $20,347(16.5)$ \\
\hline & No COPD & $76,667(74.7)$ & $25,989(25.3)$ & & $30,413(29.6)$ & $72,243(70.4)$ & & $102,656(83.5)$ \\
\hline & Pneumonia & $1561(71.3)$ & $628(28.7)$ & 0.0005 & $526(24.0)$ & $1663(76.0)$ & $<.0001$ & $2189(1.8)$ \\
\hline & No pneumonia & $90,114(74.6)$ & $30,700(25.4)$ & & $35,290(29.2)$ & $85,524(70.8)$ & & $120,814(98.2)$ \\
\hline & Diabetes & $23,734(76.7)$ & $7212(23.3)$ & $<.0001$ & $10,021(32.4)$ & $20,925(67.6)$ & $<.0001$ & $30,946(25.2)$ \\
\hline & No diabetes & $67,941(73.8)$ & $24,116(26.2)$ & & $25,795(28.0)$ & $66,262(72.0)$ & & 92,057 (74.8) \\
\hline & Arthritis & $25,744(74.3)$ & $12,372(25.7)$ & 0.116 & $13,145(27.3)$ & $34,971(72.7)$ & $<.0001$ & $48,116(39.1)$ \\
\hline & No arthritis & $55,931(74.7)$ & $18,956(25.3)$ & & $22,671(30.3)$ & $52,216(69.7)$ & & $74,887(60.9)$ \\
\hline & CRF & 9965 (74.7) & 3383 (25.3) & 0.7262 & 3730 (27.9) & 9618 (72.1) & 0.0016 & $13,348(10.9)$ \\
\hline & No CRF & $817,710(74.5)$ & $27,945(25.5)$ & & $32,086(29.3)$ & $77,569(70.7)$ & & $109,655(89.1)$ \\
\hline & ADRD & $56,261(73.8)$ & $19,941(26.2)$ & $<.0001$ & $21,548(28.3)$ & $54,654(71.7)$ & $<.0001$ & $76,202(62.0)$ \\
\hline & No ADRD & $35,414(75.7)$ & $11,387(24.3)$ & & $14,268(30.5)$ & $32,533(69.5)$ & & $46,801(38.0)$ \\
\hline & $\mathrm{HF}$ & $13,754(73.2)$ & $5041(26.8)$ & $<.0001$ & $4642(24.7)$ & $14,153(75.3)$ & $<.0001$ & 18,795 (15.3) \\
\hline & $\mathrm{NoHF}$ & 77,921 (74.8) & $26,287(25.2)$ & & $31,174(29.9)$ & $73,034(70.1)$ & & $104,208(84.7)$ \\
\hline & Cancer & 9587 (72.3) & 3677 (27.7) & $<.0001$ & 3531 (26.6) & $9733(73.4)$ & & $13,264(10.8)$ \\
\hline & No cancer & $82,088(74.8)$ & $27,651(25.2)$ & & $32,285(29.4)$ & $77,454(70.6)$ & $<.0001$ & $109,739(89.2)$ \\
\hline \multirow[t]{6}{*}{ CHESS } & 0 & 46,724 (76.9) & $14,062(23.1)$ & $<.0001$ & $19,681(32.4)$ & 41,105 (67.6) & $<.0001$ & $60,786(49.4)$ \\
\hline & 1 & $28,920(73.7)$ & $10,312(26.3)$ & & $11,075(28.2)$ & 28,157 (71.8) & & $39,232(31.9)$ \\
\hline & 2 & $11,482(71.8)$ & $4510(28.2)$ & & 3790 (23.7) & $12,202(76.3)$ & & 15,992 (13.0) \\
\hline & 3 & $3428(66.8)$ & 1701 (33.2) & & 999 (19.5) & $4130(80.5)$ & & $5129(4.2)$ \\
\hline & 4 & $1013(62.8)$ & $599(37.2)$ & & $247(15.3)$ & 1365 (84.7) & & $1612(1.3)$ \\
\hline & 5 & $108(42.9)$ & $144(57.1)$ & & $24(9.5)$ & $228(90.5)$ & & $252(0.2)$ \\
\hline \multirow{2}{*}{$\begin{array}{l}\text { Physician visit in last } \\
14 \text { days }\end{array}$} & Yes & 72,875 (74.2) & $25,302(25.8)$ & $<.0001$ & $28,777(29.3)$ & $69,400(70.7)$ & 0.003 & 98,177 (79.8) \\
\hline & No & $18,800(75.7)$ & $6026(24.3)$ & & 7039 (28.4) & $17,787(71.6)$ & & $24,826(20.2)$ \\
\hline \multirow[t]{4}{*}{ CPS } & 0 & $11,197(79.3)$ & $2932(20.7)$ & $<.0001$ & 4913 (34.8) & 9216 (65.2) & $<.0001$ & $14,129(11.5)$ \\
\hline & 1,2 & 33,974 (75.8) & $10,873(24.2)$ & & $13,413(29.9)$ & $31,434(70.1)$ & & $44,847(36.5)$ \\
\hline & 3,4 & $37,545(73.4)$ & $13,593(26.6)$ & & $14,249(27.9)$ & $36,889(72.1)$ & & $51,138(41.6)$ \\
\hline & 5,6 & 8959 (69.5) & 3930 (30.5) & & $3241(25.2)$ & 9648 (74.8) & & $12,889(10.5)$ \\
\hline \multirow[t]{3}{*}{ ADLH } & Scale $=0$ & $5036(80.4)$ & 1228 (19.6) & $<.0001$ & 2145 (34.2) & 4119 (65.8) & $<.0001$ & $6264(5.1)$ \\
\hline & Scale $=1,2$ & $25,762(77.0)$ & 7698 (23.0) & & $10,590(31.7)$ & $22,870(68.3)$ & & $33,460(27.2)$ \\
\hline & Scale $>=3$ & 60,877 (73.1) & $22,402(26.9)$ & & $23,081(27.7)$ & $60,198(72.3)$ & & $83,279(67.7)$ \\
\hline
\end{tabular}

$A B$ Alberta, $B C$ British Columbia, ON Ontario, COPD Chronic Obstructive Lung Disease, ADRD Alzheimer's Disease and Related Dementias, CRF Chronic Renal Failure, HF Heart failure, CHESS Changes in Health, End-Stage Disease, and Signs and Symptoms Scale, CPS Cognitive Performance Scale, ADLH Activities of Daily Living Hierarchy Scale 
Table 3 shows absolute event rates by presence of absence of DNR and DNH directives. Both the presence of DNR and DNH directives were associated with fewer hospitalization and higher mortality at 90 days.

\section{Association between DNR and DNH directives and health transitions for LTCH residents at 90-day follow-up $\left(\mathrm{T}_{2}\right)$}

Table 4 summarizes the association between DNR and DNH directives and subsequent health outcomes.

\section{DNH directives}

The presence of DNH directives was associated with increased odds of transition to a higher health instability level while remaining in the LTCH for residents with low or medium baseline health instability. Similarly, the odds of transitions to better health were lower for residents with medium or high baseline health instability. The presence of $\mathrm{DNH}$ directive was also associated with increased odds of dying irrespective of baseline resident health instability.
The presence of $\mathrm{DNH}$ directives was associated with decreased odds of admission to acute care hospital overall. Residents with high baseline health instability had lower odds of admission to acute care (OR 0.47; 95\% CI $0.43-0.52)$ than residents with medium and low baseline health instability (OR 0.63 ; 95\% CI $0.61-0.65$ and OR 0.67; 95\% CI 0.65-0.69 respectively).

\section{DNR directives}

The presence of DNR directives was associated with increased odds of transition to a higher health instability level while remaining in the LTCH for residents with baseline low or medium health instability. As with DNH, the odds of transition toward improved health stability was lower with the presence of a DNR for the applicable baseline levels. The presence of a DNR directive was also associated with increased odds of dying for residents with low or medium baseline health instability; however, it was non-significant at the high instability level.

The presence of DNR directives was associated with decreased odds of admission to acute care hospital for

Table 3 Percentage of residents with DNR and DNH and who were acutely hospitalized or died within 90 days of admission to a LTCH ( $n=123,003$ residents)

\begin{tabular}{|c|c|c|c|c|c|c|c|c|}
\hline & \multicolumn{4}{|c|}{ Do Not Resuscitate } & \multicolumn{4}{|c|}{ Do Not Hospitalize } \\
\hline & Not Present & Present & Difference & $\begin{array}{l}\mathrm{X}^{2} \\
p \text { value }\end{array}$ & Not Present & Present & Difference & $\begin{array}{l}\mathrm{X}^{2} \\
p \text { value }\end{array}$ \\
\hline Hospitalized & 23.2 & 18.8 & -4.4 & $<.0001$ & 22.0 & 14.5 & -7.5 & $<.0001$ \\
\hline Death & 19.4 & 29.5 & +10.1 & $<.0001$ & 24.3 & 33.0 & +8.7 & $<.0001$ \\
\hline
\end{tabular}

Table 4 Association between DNR and DNH directives and end-of-life outcomes for LTCH residents at 90-day follow-up ( $\left.T_{2}\right)$ by state of health instability at baseline

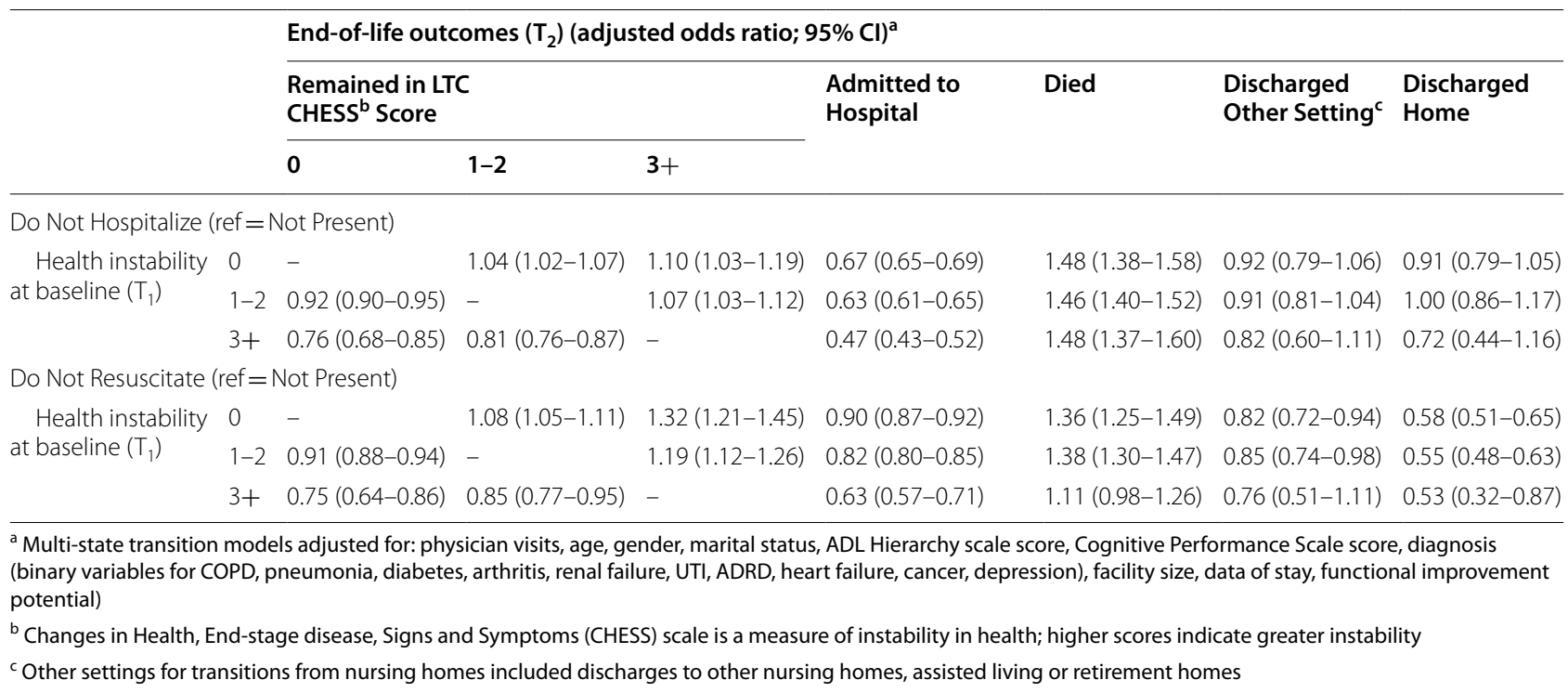


the three health instability levels at baseline. It was also associated with decreased likelihood of discharge to another setting for residents with low or medium baseline health instability. However, in presence of a DNR directive, the odds of admission to acute care hospital for residents with high baseline health instability were significantly lower (OR $0.63 ; 95 \%$ CI $0.57-0.71$ ) than the odds of admission to hospital for residents with low or medium baseline health instability (OR $0.82 ; 95 \%$ CI $0.80-0.85$ and OR $0.90 ; 95 \%$ CI $0.87-0.92$ respectively).

\section{Discussion}

In this population sample of LTCH residents in 3 Canadian provinces, more than two-thirds of residents had a DNR directive and about 1 in 4 had a DNH directive. $\mathrm{DNH}$ orders were also frequently overturned. Both care directives were associated with greater health instability and death, but lower odds of hospitalization. The effect of both directives was greater among residents with the highest baseline health instability, primarily for worsening health instability and hospitalizations.

This manuscript is the fifth in a series of articles examining health transitions among $\mathrm{LTCH}$ residents and home care clients $[2,28-30]$. Our findings that directives in LTCH are associated with lower odds of hospitalization support and further extend the generalizability of findings from recent literature [7, 8, 31]. Our use of multistate transitions models is better able than survival analysis to account for competing risks, and thus better able to model trajectories of change for multiple outcomes [2, 28-30]. Our findings also contribute to a greater understanding of the association between these directives to health instability, an outcome more specific to resident health than a health system outcome such as hospitalization.

The literature has shown that LTCH residents are frequently transferred to acute care hospitals at the end of life, a care episode from which many derive little benefit, and some may be harmed [2-4]. The intent of advance care planning is to properly understand the wishes of residents nearing the end of life, ensuring that these are well-informed with respect to the risks and benefits of interventions such as cardiopulmonary resuscitation and acute care visits. DNR and DNH directives are intended to capture the results of ACP discussions and support subsequent care planning. Our results and those of an older study by the Canadian Institute of Health Information confirm that the use DNR directives is common and has remained consistent over time [32]. The Canadian Institute of Health Information report also showed that DNR directives are rarely reversed in only $0.05 \%$ of residents. Taken together, these findings suggest that DNR directives are easily explained, universally understood, and sufficiently specific to be meaningfully adopted and implemented as part of an advance care planning process by LTCH teams, families and residents [32-34].

In contrast, our findings suggest that $\mathrm{DNH}$ directives have limitations. Even though they were associated with decreased rates of acute hospitalization, they were implemented only $26 \%$ of the time. Moreover, DNH directives were not associated with an absolute avoidance of hospitalization but with significant odds of being reversed, even among the residents with elevated baseline health instability and thus possibly at the end of life. Our findings, along with those of others who found no associations between a DNH directive and the odds of hospitalization, raise questions not only about the effectiveness of the DNH directive, but also about its acceptability and usefulness [33].

In our study, higher levels of health instability were associated with lower odds of being hospitalized among residents with either a DNR or a DNH directive in place, though these odds remain substantial. Higher baseline health instability is associated with greater odds of transition to a worsening health state, at which the risk of death is high regardless of whether a resident is hospitalized or not [2]. Other studies, however, have identified health instability as an independent predictor of hospitalization [35]. Taken together, these findings not only raise questions about whether resident wishes are fully respected during a health crisis, but also whether the advance care planning discussions that led to the $\mathrm{DNH}$ orders truly reflected well-informed resident wishes or were even person-centered. First, capable residents have the right to revise prior decisions about care and accept transfer to hospital for an acute illness $[9,36]$. This right is recognized in some jurisdictions that require that all treatment decisions, such as whether to hospitalize or not, can only be made when they are required, and not ahead of time "just in case" [9, 37]. Second, despite previously expressed and informed resident wishes, hospital transfer may be appropriate for care of concerns for which the $\mathrm{LTCH}$ is unable or ill-equipped to address adequately, such as fracture management or urinary retention $[9,36]$. Third, optimal approaches to ACP should always engage residents to elicit their perspectives and wishes, even though they may not be fully capable to decide [9]. We have previously shown that advance care planning conversations in Canadian LTCH often fail to sufficiently engage residents such that their wishes and goals are not fully expressed, nor understood by substitute decision makers or the care team $[9,38]$. Moreover, residents of $\mathrm{LTCH}$ are often not provided with adequate information about their specific health context that would help them formulate and express care wishes [9, 38]. Finally, decisions may be related to institutional policies and 
practices. For instance, emergencies are often managed by on-call physicians who may not be familiar with the resident, have restricted access to advance care planning documentation, or face a distressed and unprepared family, and therefore err on the side of a transfer to an acute care hospital $[9,38]$. Thus, while a DNH directive may be a consequential indicator of LTCH resident outcomes, it may not reflect adequate advance care planning or truly informed resident wishes. However, the CHESS scale, as a measure of a health instability that is routinely available upon admission to a LTCH, may represent important health context information to share with a resident or substitute decision maker when formulating care wishes.

This study has several strengths. First, the longitudinal cohort study design made it is easier to establish the existence of an appropriate time sequence. Second, the large sample size allowed to have less variability around the estimates and to make more reliable inferences. Third, the use of multistate transition models better accounts for competing risks and better describes resident health trajectories than regression models. There are a few limitations to consider. First, the data set did not include variables related to ethnicity, religious affiliation, facility ownership and operation, LTCH policies or practice that might shed further light on the results [39]. Similarly, we were unable to assess the quality and content of the advance care planning conversations that led to the documentation of DNH/DNR directives. Finally, additional prognostic factors that may explain some of the observed outcomes may not have been measured and considered in our analysis. However, we were able to examine a rich set of resident characteristics thus controlling for several confounding factors.

\section{Conclusion}

Even though both DNH and DNR directives were more likely to be present for LTCH residents at risk of transition to greater health instability and death, DNH directives were present for only $26 \%$ residents and were frequently overturned, even for the most unstable residents. Our findings raise concerns that the DNH directive many be less aligned with resident wishes than it is reflective of a desired health service outcome. In light of our findings, and given emerging ethical concerns in some jurisdictions, we suggest that policies recommending DNH directives as a suitable indicator of resident-centered advance care planning be re-evaluated. Our findings also suggest the need to improve advance care planning in Canadian LTCH in a manner that enables residents to fully express their values and wishes in the context of their individual medical realities, supports person-centered decision-making at the point of care, and ensures that LTCH have the resources to deliver this care. The CHESS scale, as a measure of health instability, may be of value to inform advance care planning discussions with residents and their substitute decision makers.

\begin{abstract}
Abbreviations
AB: Alberta; ADLH: Activities of Daily Living Hierarchy Scale; ADRD: Alzheimer's Disease and Related Dementias; BC: British Columbia; CCRS: Complex Continuing Care Reporting System; CHESS: Changes in Health, End-Stage Disease, Signs, and Symptoms Scale; COPD: Chronic Obstructive Lung Disease; CPS: Cognitive Performance Scale; CRF: Chronic Renal Failure; DAD: Discharge Abstract Database; DNR: Do-not-resuscitate; DNH: Do-not-hospitalize; HF: Heart failure; LTC: long-term care; LTCH: long-term care home; ON: Ontario; RAI-MDS: InterRAI Resident Assessment Instrument-Minimum Data Set.
\end{abstract}

\section{Acknowledgements}

The authors thank Jonathan Chen and Micaela Jantzi for their work on the data analysis as well as Julie Koreck, Nisreen Murad, and Marie-Noël Nadeau for administrative assistance, all of whom have provided written consent to be thus recognized.

\section{Authors' contributions}

GH confirms that all authors listed contributed significantly according to authorship guidelines. JH, $\mathrm{PH}, \mathrm{GH}$ and $\mathrm{AC}$ all contributed to the study design. $\mathrm{JH}$ led the data analysis. All authors contributed to data interpretation. RA and GH led the drafting of the manuscript, with all others contributing substantively and approving the final version.

\section{Funding}

This study was funded by a Strategic Impact Grant from the Canadian Frailty Network (SIG2014F-31). George Heckman is supported by the Schlegel Chair in Geriatric Medicine at McMaster University. Andrew Costa is supported by the Schlegel Chair in Clinical Epidemiology and Aging and McMaster University. None of the funding sources played a role in the design, methods, subject recruitment, data collections, analysis and preparation of paper.

\section{Availability of data and materials}

The datasets used and/or analysed during the current study are available from the corresponding author on reasonable request.

\section{Declarations}

Ethics approval and consent to participate

We obtained research ethics approval from the University of Waterloo's Office of Research Ethics (ORE no. 18228). This is secondary data analysis of clinical data so we did not obtain approval from individuals. Use of the CCRS data is authorized under UW ORE no. 30372 (Secondary Analyses of Anonymized interRAl Data from the Data Sharing Agreement with the Canadian Institute for Health Information), stating that this represents secondary data analysis of assessments collected as part of normal clinical practice and anonymized before being transmitted to the University of Waterloo.

\section{Consent for publication}

Not applicable.

\section{Competing interests}

The authors declare that they have no competing interests.

\section{Author details}

${ }^{1}$ Carrefour de l'innovation et de l'évaluation en santé, Centre Hospitalier de I'Université de Montréal, Montréal, Canada. ${ }^{2}$ School of Public Health Sciences, University of Waterloo, 200 University Avenue West, Waterloo, Ontario N2L 3G1, Canada. ${ }^{3}$ Schlegel Research Institute for Aging, Waterloo, Canada. ${ }^{4}$ McMaster University, Hamilton, Canada. 
Received: 3 December 2020 Accepted: 6 December 2021

Published online: 03 January 2022

\section{References}

1. Hirdes JP, Mitchell L, Maxwell CJ, White N. Beyond the 'iron lungs of gerontology': using evidence to shape the future of nursing homes in Canada. Can J Aging. 2011;30:371-90. https://doi.org/10.1017/S0714 980811000304

2. Hirdes JP, Heckman GA, Morinville A, et al. One Way Out? A Multistate Transition Model of Outcomes After Nursing Home Admission. J Am Med Dir Assoc. 2019;20:1425-1431.e1. https://doi.org/10.1016/j.jamda. 2019.02.010.

3. Canadian Institute of Health Information. Profile of residents in residential and hospital-based continuing care 2017-2018. Ottawa: CIHI; 2018.

4. Canadian Institute for Health Information. Sources of potentially avoidable emergency department visits. Ottawa: CIHI; 2014.

5. Rahemi Z, Williams CL. Older adults of underrepresented populations and their end-of-life preferences: an integrative review. ANS Adv Nurs Sci. 2016;39:E1-E29. https://doi.org/10.1097/ANS.0000000000000148.

6. Sudore RL, Lum HD, You JJ, et al. Defining advance care planning for adults: a consensus definition from a multidisciplinary delphi panel. J Pain Symptom Manage. 2017;53:821-32.e1. https://doi.org/10.1016/j. jpainsymman.2016.12.331.

7. Brinkman-Stoppelenburg A, Rietjens JA, van der Heide A. The effects of advance care planning on end-of-life care: a systematic review. Palliat Med. 2014;28:1000-25. https://doi.org/10.1177/0269216314526272.

8. Tanuseputro P, Hsu A, Chalifoux M, et al. Do-not-resuscitate and donot-hospitalize orders in nursing homes: who gets them and do they make a difference? J Am Med Dir Assoc. 2019;20:1169-1174.e1. https:// doi.org/10.1016/j.jamda.2019.02.017.

9. Heckman GA, Boscart V, Keller H, et al. Applying the knowledge-toaction framework to engage stakeholders and solve shared challenges with person-centered advance care planning in long-term care homes. Can J Aging. 2021;15:1-11. https://doi.org/10.1017/S07149808200004 10 Epub ahead of print. PMID: 33583447.

10. Benchimol El, Smeeth L, Guttmann A, Harron K, Moher D, Petersen I, et al. The REporting of studies conducted using observational routinely-collected health data (RECORD) statement. Plos Med. 2015;12(10):e1001885.

11. von Elm E, Altman DG, Egger M, Pocock SJ, Gotzsche PC, Vandenbroucke $J P$, et al. Strengthening the reporting of observational studies in epidemiology (STROBE) statement: guidelines for reporting observational studies. BMJ. 2007;335(7624):806-8.

12. Hirdes JP, Poss JW, Caldarelli $\mathrm{H}$, et al. An evaluation of data quality in Canada's continuing care reporting system (CCRS): secondary analyses of Ontario data submitted between 1996 and 2011. BMC Med Inform Decis Mak. 2013;13:27. https://doi.org/10.1186/ 1472-6947-13-27.

13. Juurlink D, Preyra C, Croxford R, Chong A, Austin P, Tu J, et al. Canadian Institute for Health Information Discharge Abstract Database: A Validation Study: Institute for Clinical Evaluative Sciences; 2006. https://www. ices.on.ca/ /media/Files/Atlases-Reports/2006/CIHI-DAD-a-validationstudy/Full-report.ashx (Access verified June 16, 2021)

14. Canadian Institute of Health Information. Data Quality Documentation, National Ambulatory Care Reporting System - Current-Year Information, 2019-2020. https://www.cihi.ca/sites/default/files/document/ nacrs-data-quality-current-year-information2019-2020-en.pdf (Access verified June 16, 2021).

15. Morris JN, Carpenter I, Berg K, Jones RN. Outcome measures for use with home care clients. Can J Aging. 2000;19:87-105

16. Hirdes JP, Frijters DH, Teare GF. The MDS-CHESS scale: a new measure to predict mortality in institutionalized older people. J Am Geriatr Soc. 2003;51:96-100. https://doi.org/10.1034/j.1601-5215.2002.51017.x.

17. Hirdes JP, Poss JW, Mitchell L, Korngut L, Heckman G. Use of the interRAI CHESS scale to predict mortality among persons with neurological conditions in three care settings. PLoS One. 2014;9:e99066. https://doi. org/10.1371/journal.pone.0099066.

18. Burrows AB, Morris JN, Simon SE, Hirdes JP, Phillips C. Development of a minimum data set-based depression rating scale for use in nursing homes. Age Ageing. 2000;29:165-72. https://doi.org/10.1093/ageing/ 29.2.165.

19. Szczerbińska K, Hirdes JP, Życzkowska J. Good news and bad news: depressive symptoms decline and undertreatment increases with age in home care and institutional settings. Am J Geriatr Psychiatry. 2012;20:1045-56. https://doi.org/10.1097/JGP.0b013e3182331702.

20. Landi F, Tua E, Onder G, et al. Minimum data set for home care: a valid instrument to assess frail older people living in the community. Med Care. 2000:38:1184-90. https://doi.org/10.1097/00005650-20001 2000-00005

21. Morris JN, Fries BE, Mehr DR, et al. MDS cognitive performance scale@. J Gerontol. 1994;49:M174-82. https://doi.org/10.1093/geronj/49.4. m174.

22. Morris JN, Howard EP, Steel K, et al. Updating the cognitive performance scale. J Geriatr Psychiatry Neurol. 2016;29:47-55. https://doi. org/10.1177/0891988715598231.

23. Hirdes JP, Fries BE, Morris JN, et al. Integrated health information systems based on the RAI/MDS series of instruments. Healthc Manage Forum Winter 1999;12(4):30-40.

24. Hirdes JP, Ljunggren G, Morris JN, et al. Reliability of the interRAl suite of assessment instruments: a 12-country study of an integrated health information system. BMC Health Serv Res. 2008;8:277.

25. Poss JW, Jutan NM, Hirdes JP, et al. A review of evidence on the reliability and validity of minimum data set data. Healthc Manage Forum. 2008;21:33-9.

26. Cook RJ, Berg K, Lee K-A, Poss JW, Hirdes JP, Stolee P. Rehabilitation in home care is associated with functional improvement and preferred discharge. Arch Phys Med Rehabil. 2013;94:1038-47. https://doi.org/10. 1016/j.apmr.2012.12.024.

27. Cook RJ, Lawless JF. Multistate models for the analysis of life history data: CRC Press; 2018.

28. Heckman GA, Hirdes JP, Hébert PC, et al. Predicting future health transitions among newly admitted nursing home residents with heart failure. J Am Med Dir Assoc. 2019;20:438-43. https://doi.org/10.1016/j. jamda.2018.10.031

29. Hébert PC, Morinville A, Costa A, Heckman G, Hirdes J. Regional variations of care in home care and long-term care: a retrospective cohort study. CMAJ Open. 2019;7:E341-50. https://doi.org/10.9778/cmajo. 20180086

30. Heckman GA, Hirdes JP, Hébert $P$, et al. Assessments of heart failure and frailty-related health instability provide complementary and useful information for home care planning and prognosis. Can J Cardiol. 2021;S0828-282X(21):00374-3. https://doi.org/10.1016/j.cjca.2021.07. 009 Epub ahead of print. PMID: 34303783.

31. Gozalo P, Teno JM, Mitchell SL, et al. End-of-life transitions among nursing home residents with cognitive issues. N Engl J Med. 2011;365:1212-21. https://doi.org/10.1056/NEJMsa1100347.

32. Canadian Institute for Health Information. A snapshot of advance directives in long-term care: how often is "do not" done? Ottawa: $\mathrm{ClHI}$; 2016.

33. Anderson JE, Sikorski I, Finucane TE. Advance care planning by or on behalf of peritoneal dialysis patients in long-term care. Am J Kidney Dis. 2006:48:122-7. https://doi.org/10.1053/j.ajkd.2006.03.041.

34. Finucane TE, Denman SJ. Deciding about resuscitation in a nursing home theory and practice. J Am Geriatr Soc. 1989;37:684-8. https:// doi.org/10.1111/j.1532-5415.1989.tb02228.x.

35. Kadu M, Heckman GA, Stolee P, Perlman C. Risk of hospitalization in long-term care residents living with heart failure: a retrospective cohort study. Can Geriatr J. 2019;22:171-81. https://doi.org/10.5770/ cgj.22.366.

36. Cohen AB, Knobf MT, Fried TR. Do-not-hospitalize orders in nursing homes: "call the family instead of calling the ambulance". J Am Geriatr Soc. 2017;65:1573-7. https://doi.org/10.1111/jgs.14879.

37. Wahl JA, Dykeman MJ, Walton T. Health care consent, advance care planning, and goals of care practice tools: the challenge in Getting it right. In: Improving the Last Stages of Life, Commissioned by the Law Commission of Ontario; 2016. Accessed at http://www.advocacyce ntreelderly.org/appimages/file/ACE\%20DDO\%20Walton\%20Formatted\%20Dec\%202,2016\%20LCO.pdf August 17, 2020.

38. Choi N, Garland A, Keller H, et al. Problems with Advance Care Planning Processes and Practices in Nursing Homes. J Am Med Dir Assoc. 
2020;21(12):2012-3. https://doi.org/10.1016/j.jamda.2020.07.010 Epub 2020 Aug 18. PMID: 32826160; PMCID: PMC7434431.

39. Office of the Seniors Advocate of British Columbia. From Residential Care to Hospital: an Emerging Pattern. 2018. Accessed at https://www. seniorsadvocatebc.ca/app/uploads/sites/4/2018/08/ResidentialCare toHospital-report.pdf November 20, 2021.

\section{Publisher's Note}

Springer Nature remains neutral with regard to jurisdictional claims in published maps and institutional affiliations.

- fast, convenient online submission

- thorough peer review by experienced researchers in your field

- rapid publication on acceptance

- support for research data, including large and complex data types

- gold Open Access which fosters wider collaboration and increased citations

- maximum visibility for your research: over 100M website views per year

At BMC, research is always in progress.

Learn more biomedcentral.com/submissions 